\title{
140. On the Mechanism of the Change of Space-lattice in Different Modifications of Crystals.
}

By Hikoroku SHôjI.

(Comm. by S. Nakamura, M.I.A., Oct. 12, 1929.)

I. It is assumed that, when a space-lattice of one modification of a substance is freely and continuously changed into that of another, the atoms or molecules which are most firmly bound together in the lattice have the greatest tendency to move always in groups during the change. Now the atoms or molecules are most firmly bound together in the planes of the greatest atomic or molecular cohesion of the lattice. Thus it is very probable that the atoms or molecules in the planes of the greatest atomic or molecular cohesion of the lattice of one modification of a substance always move in groups during the transformation and these planes transform also into those of another modification and vice versa. On examining the transformations of crystals we find that, this is actually the case when the transformation can occur indifferently in either direction with great velocity, as in the cases of $\mathrm{Fe}, \mathrm{Co}, \mathrm{NH}_{4} \mathrm{Cl}, \mathrm{NH}_{4} \mathrm{Br}, \mathrm{NH}_{4} \mathrm{I}$ and $\mathrm{AgI}$. For instance the transformation of the lattice of iron can occur through the slip- or shear-like deformation along the octahedral planes of the face-centred cubic lattice or along the rhombic dodecahedral planes of the bodycentred cubic lattice, the four pairs of the octahedral planes of the former and the four pairs of the rhombic dodecahedral planes of the latter changing into each other completely in a reversible manner.

II. The change of lattice comes from the change in atoms or molecules themselves. If in this case the increase of the atomic or molecular force, for example, in a special direction of the lattice is particularly great, then it is possible that the plane of the greatest cohesion of one modification does not change into that of the other. For instance the octahedral plane of the grey tin which is the plane of the greatest cohesion of its lattice does not change by the transformation into the plane of the greatest cohesion of white tin. Now as the transformation between grey and white tin can occur through the slip-like deformation along the planes of the greatest cohesion of the former, but not along those of the latter, grey tin can transform into white, but not white tin into grey with great velocity. 
The transformation can thus occur also through the slip-like deformation of the lattice along the planes other than those of the greatest cohesion but with a very small velocity. If the transformation cannot occur in one direction or in both directions through the slip-like deformation of the lattice only along the planes of the greatest cohesion, then the transformation can occur only with a small velocity in the one direction or in both directions. From this point of view, we can understand that the different modifications of $\mathrm{C}, \mathrm{TiO}_{2}, \mathrm{ZnS}$ and $\mathrm{CaCO}_{3}$ can exist in nature at the same time.

III. In the lattice of some of the optically active substances some kind of atoms are distributed in a spring-or screw-like manner and are firmly bound spring-like together. Such a lattice would by the transformation be deformed like a spring -i. e. with a great deal of chang of length in one direction and a very small change in the perpendicular directions. The velocity of this transformation must be very small, because the spring-like motion of the atoms in the lattice is very complicated and the resistance to the motion should be very great. The transformation between $\beta$-quartz and $\beta$-cristobalite and that between cinnabar and metacinnabar are of this kind, where $\beta$-quartz and cinnabar are optically active. The change of length which takes place in the former is to the extent of about 13 per cent of the length of the lattice of $\beta$-quartz in one direction and 0.4 per cent in the perpendicular directions, while in the latter, it is to the extent of about 11 per cent of the length of the lattice of cinnabar in one direction and 0.5 per cent in the perpendicular directions.

IV. Some substances such as $\mathrm{C}, \mathrm{SiO}_{2}$ or $\mathrm{SiC}$ have a so-called amorphous modification. This suggests that the motion of the atoms or molecules of such a substance is exceedingly slow, because the amorphous is not the most stable form. It follows that the velocity of the transformation of the crystal-modification of that substance is small.

V. The transformations above considered are due to a great or a complicated change of lattice corresponding to the plastic deformation of the body. Then there might exist transformations which are due to a small or a simple change of lattice corresponning to the elastic deformation of the body. In the latter case the lattice of one modification should be a very slight distortion of that of the other and the transformation between those could generally occur in both directions with great velocity. The transformation between $\alpha$ - and $\beta$ quartz belongs to this class. 
The above description of the mechanism of the change of lattice is strictly applicable to the smallest part of the lattice. Such changes can occur in different parts of the lattice in different directions at the same time according to the degree of symmetry of the lattice.

Thanks are due to Prof. $K$. Honda for his kind guidance and to Prof. M. Watanabe for his useful advice. 